\title{
Finding novel pyrophosphate-dependent kinases based on their donor selectivity determinants revealed by crystal structures
}

\author{
Masahiro Fujihashi, ${ }^{1}$ Ryuhei Nagata, ${ }^{1}$ Takaaki Sato, ${ }^{2}$ Haruyuki Atomi ${ }^{2}$ \& Kunio Miki ${ }^{1}$ \\ ${ }^{1}$ Department of Chemistry, Graduate School of Science, Kyoto University, Sakyo-ku, \\ Kyoto 606-8502, Japan \\ 2 Department of Synthetic Chemistry and Biological Chemistry, Graduate School of \\ Engineering, Kyoto University, Katsura, Nishikyo-ku, Kyoto 615-8510, Japan
}

\begin{abstract}
Almost all kinases use ATP as their phosphate donor, while a few kinases utilize inorganic pyrophosphate (PPi) instead. PPi-dependent kinases are often homologous to their ATPdependent counterparts, but determinants of their different donor specificities remain unclear. We identify a PPi-dependent member of the ribokinase family. Determination of its crystal structures complexed with PPi analogs elucidates the PPi-binding mode of this enzyme. Structural comparison and sequence alignment reveal five important residues: three basic residues specifically recognizing PPi and two large hydrophobic residues occupying a part of the ATP-binding pocket. Two of the three basic residues adapt a conserved motif of the ribokinase family for the PPi binding. Using these five key residues collectively as a signature pattern, we discover novel PPi-specific members of the ribokinase family. Introduction of these residues may enable transformation of ATPdependent ribokinase family members into PPi-dependent enzymes.
\end{abstract}

\section{Reference}

1. Nagata, R., Fujihashi, M., Sato, T., Atomi, H. \& Miki, K. Nat. Commun. accepted. 